Research Article

\title{
Clinical Profile and Subtypes of Glaucoma in a District Hospital in North Goa
}

\author{
Sparsh Naik', Jagadish Cacodcar', Saleena Naik ${ }^{3}, \underline{\text { Tanvi Poy Raiturcar }}^{4}$
}

${ }^{1}$ M.B.B.S Student (Third Year Part-1), Goa Medical College and Hospital, Bambolim, Goa, India.

${ }^{2}$ Professor and Head, Department of Preventive and Social Medicine, Goa Medical College and Hospital, Bambolim, Goa, India.

${ }^{3}$ Senior Ophthalmic Surgeon, Asilo Hospital Mapusa, Mapusa, Goa, India.

${ }^{4}$ Senior Resident, Department of Ophthalmology, Goa Medical College and Hospital, Bambolim, Goa, India.

DOI: https://doi.org/10.24321/2455.7048.202022

\section{I $\quad \begin{array}{lllll}\mathbf{N} & \mathbf{F} & \mathbf{O}\end{array}$}

\section{Corresponding Author:}

Dr. Tanvi Poy Raiturcar, Hno 992, St. Joaquim Road Borda, Goa, India.

E-mail Id:

tanvi1491@gmail.com

Orcid Id:

https://orcid.org/0000-0001-8910-9982

How to cite this article:

Naik S, Cacodcar J, Naik S, Raiturcar TP. Clinical Profile and Subtypes of Glaucoma in a District Hospital in North Goa. Epidem Int 2020; 5(3): 15-18.

Date of Submission: 2020-05-12

Date of Acceptance: 2020-08-20

\section{$\begin{array}{llllllll}\mathbf{A} & \mathbf{B} & \mathbf{S} & \mathbf{T} & \mathbf{R} & \mathbf{A} & \mathbf{C} & \mathbf{T}\end{array}$}

Background: Glaucoma, a leading cause of blindness in the world, has a global burden of 64.3 million. Studies in other States in India have described the clinical profile of glaucoma. However, no such study was conducted in Goa. Hence, our study was undertaken to identify and the clinical profile and subtypes of glaucoma in Goa.

Methodology: A prospective hospital-based study was conducted over 6 months among patients attending Ophthalmology OPD at the North Goa District Hospital. The study participants underwent a detailed Ophthalmological evaluation by the senior Ophthalmic surgeon. The data so collected was entered in pre-tested semi-structured proformas. Statistical analysis was done using SPSS version 22.

Result: Most glaucoma patients were females (62.5\%) and elderly (60.71\%). The most common glaucoma was primary angle closure glaucoma (58.93\%), followed by primary open angle glaucoma and glaucoma suspects. Majority patients had associated risk factors such as diabetes, hypertension and family history of glaucoma.

Keywords: Glaucoma, Clinical Profile, Sub-Types, Goa

\section{Introduction}

Glaucoma is a leading cause of blindness in the world, with a global prevalence of $3.54 \%$. Currently there are 64.3 million cases of glaucoma in the world which is expected to increase 111.8 million by the year $2040 .{ }^{1} \mathrm{It}$ is worthwhile to note that one in eight individuals in India aged more than 40 years is either suffering from or is at risk of development of glaucoma. ${ }^{2}$ India currently accounts for $12.9 \%$ of the cases of Primary open angle glaucoma and $12.7 \%$ cases of primary angle closure glaucoma in the world. ${ }^{3}$ There have been many studies done in other Indian states to describe the profile of glaucoma. ${ }^{4,5,6}$ however, such a study has been lacking in Goa. Through this study we have described the clinical profile of glaucoma patients presenting at the district hospital in North Goa.

\section{Aims and Objectives}

- To describe the clinical profile glaucoma patients presenting to the district hospital in North Goa

- To identify the risk factors associated with glaucoma

\section{Materials and Methods}

Our case-series study was conducted at the North Goa district hospital during the time period from April to September 2019. A total of 56 patients were included in 
our study by the census method of sampling. The patients underwent a detailed clinical evaluation by the senior ophthalmic surgeon at the hospital. Ethical approval was obtained from the Ethics committee of the Directorate of Health services, Goa. Informed Consent of the study participants was duly obtained.

The study participants comprised of individuals who were diagnosed with glaucoma or were glaucoma suspects. These included patients having optic nerve head changes suggestive of glaucoma, visual field defects specific to glaucoma or those having intraocular pressure more than $21 \mathrm{~mm} \mathrm{Hg}$. The patients, who did not have optic nerve head changes, raised intraocular pressure or visual field changes suggestive of glaucoma were excluded from our study. The participants were subjected to a detailed workup which included a detailed history, visual acuity, slit-lamp examination, gonioscopy, optic nerve head evaluation, peripheral fundus examination and Humphrey field analysis. The data was entered into Microsoft excel 2010 and analyzed using SPSS statistical software version 22 .

\section{Result}

In our study we evaluated 56 consecutive glaucoma patients who had presented to the North Goa District hospital during a period of 6 months from April 2019 to September 2019. Out of the 56 patients majority, i.e., 35 (62.5\%) were females and the rest were males (Table 1). Most were elderly patients aged between 60 to 70 years, i.e., $34(60.71 \%)$, whereas $10(17.85 \%)$ were between 50 to 60 years (Table 2). The most common type of glaucoma in our study population was primary angle closure glaucoma, i.e., 33 participants (58.92\%); followed by primary open angle glaucoma, i.e., 12 (21.43\%); glaucoma suspects, i.e., 9 (16.07\%); secondary glaucoma, i.e., 1 (1.78\%) and combined mechanism glaucoma 1 (1.78\%) (Table 3$)$.

Table I.Sex distribution of glaucoma patients

\begin{tabular}{|c|c|c|}
\hline Type of Glaucoma & $\begin{array}{c}\text { Males No. } \\
\text { (\%) }\end{array}$ & $\begin{array}{c}\text { Females } \\
\text { No. (\%) }\end{array}$ \\
\hline Suspect & $3(5.35 \%)$ & $6(10.71 \%)$ \\
\hline $\begin{array}{c}\text { Primary angle closure } \\
\text { glaucoma }\end{array}$ & $14(25 \%)$ & 19 (33.92\%) \\
\hline $\begin{array}{l}\text { Primary open angle } \\
\text { glaucoma }\end{array}$ & $4(7.14 \%)$ & $8(14.28 \%)$ \\
\hline Normal tension glaucoma & $0(0 \%)$ & $0(0 \%)$ \\
\hline Secondary glaucoma & $0(0 \%)$ & $1(1.78 \%)$ \\
\hline $\begin{array}{c}\text { Combined mechanism } \\
\text { glaucoma }\end{array}$ & $0(0 \%)$ & $1(1.78 \%)$ \\
\hline Total & $21(37.5 \%)$ & $35(62.5 \%)$ \\
\hline
\end{tabular}

Many of the patients had some associated risk factors. Ten participants (17.85\%) had coexisting diabetes, 16 participants (28.57\%) had associated hypertension, myopia was noted in 4 participants (7.14\%), history of smoking in 1 participant $(1.78 \%)$ and family history of glaucoma in 4 participants (7.14\%) (Figure 1). Majority of the participants, i.e., $45(80.35 \%)$ had a good best corrected visual acuity in the range between 6/6-6/12.

Table 2.Age distribution of study participants

\begin{tabular}{|c|c|}
\hline Age distribution (years) & Number (percentage) \\
\hline $20-30$ & $2(3.57 \%)$ \\
\hline $30-40$ & $4(7.14 \%)$ \\
\hline $40-50$ & $6(10.71 \%)$ \\
\hline $50-60$ & $10(17.85 \%)$ \\
\hline $60-70$ & $34(60.71 \%)$ \\
\hline Total & $56(100 \%)$ \\
\hline
\end{tabular}

Table 3.Distribution of glaucoma patients

\begin{tabular}{|c|c|}
\hline Type of Glaucoma & $\begin{array}{c}\text { Number } \\
\text { (percentage) }\end{array}$ \\
\hline Glaucoma suspects & $9(16.07 \%)$ \\
\hline Primary angle closure glaucoma & $33(58.92 \%)$ \\
\hline Primary open angle glaucoma & $12(21.42 \%)$ \\
\hline Normal tension glaucoma & $0(0 \%)$ \\
\hline Secondary glaucoma & $1(1.78 \%)$ \\
\hline Combined mechanism glaucoma & $1(1.78 \%)$ \\
\hline Total & $56(100 \%)$ \\
\hline
\end{tabular}

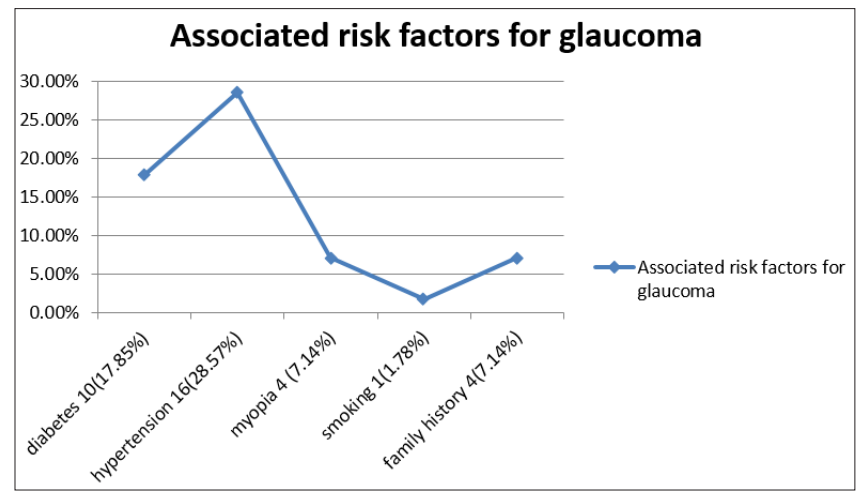

Figure I.Distribution of risk factors associated with glaucoma

\section{Discussion}

Glaucoma is a leading cause of irreversible blindness worldwide and is the third most important cause of blindness after cataract and refractive errors. ${ }^{7}$ It is estimated that there are around 3 million individuals who became blind due to glaucoma. ${ }^{7}$ India accounts for one-fifth of the global burden of glaucoma with an estimated 12 million individuals suffering from glaucoma. ${ }^{3}$ Although extensive research has been done in this field and there are many 
newer modalities of early detection and management of glaucoma, the condition still remains the leading silent killer of sight. The loss of visual acuity and visual field caused by glaucoma cannot be reversed. Through our study, we have tried to describe the clinical profile of glaucoma in our State.

In our study, we evaluated 56 consecutive patients who were known cases of glaucoma or were glaucoma suspects. We found that the most common type of glaucoma in our study population was primary angle closure glaucoma among 33 (58.92\%), followed by primary open angle glaucoma $12(21.42 \%)$, glaucoma suspects $9(16.07 \%)$, secondary glaucoma 1 (1.78\%) and combined mechanism glaucoma $1(1.78 \%)$. Our study findings are similar to the findings of a similar study conducted in North India by Das J et al., ${ }^{8}$ where the most common type of glaucoma in the study population was primary angle closure glaucoma (36.62\%) followed by glaucoma suspects, primary open angle glaucoma and the other types of glaucoma comprised a small percentage. Similar findings were reported by Sihota et al. ${ }^{9}$ and Mehta et al. ${ }^{4}$ The only case of secondary glaucoma that we found in our study was secondary to pseudoexfoliation.

Most of the participants in our study were females, i.e., 35 (62.5\%) while 21 (37.5\%) were males. This finding is consistent with that reported by Sihota et al. ${ }^{9}$ and Mehta et al. ${ }^{4}$ in similar studies. Most of the participants in our study were elderly of the age between 60 and 70 years, i.e., $34(60.71 \%)$, followed by those between 50 and 60 years i.e. $10(17.85 \%), 40$ to 50 years i.e. $6(10.71 \%), 30$ to 40 years i.e. 4 (7.14\%), and 20 to 30 years i.e. 2 (3.57\%). The reason behind this being that glaucoma is mainly a disease of the older age group. In studies by Mehta et al., ${ }^{4}$ Das J et al. ${ }^{8}$ and other such studies, ${ }^{10}$ similar findings were noted, where majority of the study population was more than 50 years of age. From this study finding, it is emphasized that there should be a high index of suspicion among the elderly patients who come with symptoms or signs pointing towards glaucoma and the necessary screening tests should be conducted.

Majority of the study participants had some associated risk factors such as diabetes, i.e., 10 (17.85\%); hypertension i.e. $16(28.57 \%)$, myopia i.e.4 (7.14\%), history of smoking i.e. $1(1.78 \%)$ and family history i.e. 4 (7.14\%). Similar results have been reported by Mehta et al ${ }^{4}$ and Sharma et al. ${ }^{10}$ in their studies.

In our study we found that the Snellen's visual acuity of majority of the participants i.e. $45(80.35 \%)$ was better than $6 / 12$, followed by $5(8.92 \%)$ participants whose vision was between $6 / 60$ and $6 / 18$, while 3 participants i.e. $5.35 \%$ patients had no perception of light. These three patients had lost vision to glaucomatous optic atrophy and could not even perceive light. An explanation for this is that glaucoma is asymptomatic till very late stages. By this time extensive irreversible damage has already occurred in terms of visual acuity and visual field loss. Another reason for this could be lack of awareness and healthcare facilities in the rural areas.

\section{Conclusion}

In this hospital based study conducted at a District hospital in North Goa we found that majority of the participants were females in the age group of more than 60 years. The most common type of glaucoma was primary angle closure glaucoma followed by glaucoma suspects and open angle glaucoma. Most patients had presented to an ophthalmologist when irreversible visual loss had already occurred. Hence, there is a need to create awareness about glaucoma among the masses so that the disease can be detected earlier and irreversible damage can be prevented with appropriate management. Family screening for glaucoma should be a very important strategy for early detection of glaucoma. Healthcare professionals at the primary healthcare level should also be trained in detection of glaucoma suspects to ensure screening from the grassroot level of the health-care system, particularly among the elderly.

\section{Conflict of Interests: None \\ References}

1. Tham YC, Li X, Wong TY, Quigley HA, Aung T, Cheng CY. Global prevalence of glaucoma and projections of glaucoma burden through 2040: a systematic review and meta-analysis. Ophthalmology 2014; 121(11): 2081-2090.

2. Ronnie G, Ve RS, Velumuri L, Asokan R, Vijaya L. Importance of population-based studies in clinical practice. Indian J Ophthalmol 2011; 59(Suppl): S1:S11-8.

3. Das J, bhomaj S, Chaudhuri Z, Sharma P et al. Profile of glaucoma in a major eye hospital in North India.Indian J ophthalmol 2001; 49(1): 25-30.

4. Quigley HA, Broman AT. The number of people with glaucoma worldwide in 2010 and 2020. Br J Ophthalmol 2006; 90: 262-267.

5. Mehta M, Mehta S, Bajaj S. Clinical profile, subtypes and risk factors among glaucoma patients in a tertiary hospital in central India. International J Scientific study 2017; 4(11): 107-112.

6. Gogoi T, Bhattacharjee S. A hospital based study of scenario of glaucoma patients in upper Assam, India. International J Research in Med sci 2017; 5(2): 607-611.

7. Sharma S, Gupta K, Kaur P et al. Clinical profile and subtypes of glaucoma in Northern India. Scholars Academic J Biosciences 2015; 3(9): 766-773.

8. Pascolini D, Mariotti SP. Global estimates of visual impairment: 2010. Br J Ophthalmol 2012; 96: 614-618.

9. Das J, bhomaj S, Chaudhuri Z, Sharma P et al. Profile of glaucoma in a major eye hospital in North India.Indian 
J ophthalmol 2001; 49(1): 25-30.

10. Sihota R, Agarwal HC. Profile of the subtypes of angle closure glaucoma in a tertiary care hospital in North India. Indian J Ophthalmol 1998; 46: 25-29.

11. Sharma S, Gupta K, Kaur P, Kaur I, Kulshrestha MR, Aggarwal A. Clinical profile and subtypes of glaucoma in Northern India. Sch Acad J Biosci 2015; 3(9): 766-773. 